\title{
Surrogate endpoints: when to use and when not to use? A critical appraisal of current evidences
}

\author{
Vinicius PEDRAZZI(a) iD \\ Fellipe Augusto Tocchini de \\ FIGUEIREDO(a) iD \\ Larisse Eduardo ADAMI(a) \\ Flávia FURLANETO(b) \\ Daniela Bazan PALIOTO(b) \\ Michel Reis MESSORA ${ }^{(b)}$ \\ (a) Universidade de São Paulo - USP, School \\ of Dentistry of Ribeirão Preto, Department \\ of Dental Materials and Prosthesis, Ribeirão \\ Preto, SP, Brazil. \\ (b) Universidade de São Paulo - USP, School \\ of Dentistry of Ribeirão Preto, Department \\ of Oral \& Maxillofacial Surgery and \\ Periodontology, Ribeirão Preto, SP, Brazil.
}

Declaration of Interests: The authors certify that they have no commercial or associative interest that represents a conflict of interest in connection with the manuscript.

\section{Corresponding Author:}

Prof. Dr. Vinicius Pedrazzi

E-mail: pedrazzi@forp.usp.br

hitps://doi.org/10.1590/1807-3107bor-2020.vol34.0074

Submitted: September 2, 2019

Accepted for publication: September 22, 2019 Last revision: June 15, 2020

\begin{abstract}
Clinical research needs to formulate a question, which must be answered by obeying ethical precepts with well-defined inclusion/ exclusion criteria and approval of the study on platforms of ethical appreciation and clinical trial records. In comparing the results or clinically relevant outcomes should be prioritized in the study of techniques, products, inputs, drugs and therapies. However, it is not always possible to use long study drawings, with many participants, and with many costs, then look for study designs with surrogate outcomes, usually a shorter path, with less sample size and considerably lower costs to the research, with shorter intervention time. Considering these outcomes as major challenges in clinical research, the premise of this work was to examine in relevant research platforms, studies on the feasibility of using surrogate endpoints for clinically relevant parameters in dentistry, with a critical evaluation of the advantages, disadvantages, and need for validation of substitute parameters for clinical studies. After a critical analysis of the results, it could be concluded that surrogate endpoints may have an important role in the initial process of developing new drugs, faster, with less sampling, and lower risk of side effects for the patient. Careful use of the surrogate endpoints is advised because, even if validated, they can provide ambiguous evidence and not be extrapolated to other populations, and may lead to bias due to the individual interpretation of each researcher. The use of unplanned surrogate outcomes that arise during the study requires a lot of caution.
\end{abstract}

Keywords: Dentistry; Clinical Trials; Ethics; Biomarkers.

\section{Introduction}

The most current concept of health is "the perfect and harmonious physical, mental, social and spiritual well-being, not simply the absence of disease"1,1,3 The wide dissemination of scientific results should be encouraged so that the benefits from the knowledge generated by the research groups could reach the common public, according to John Ziman's precepts with his post-academic science. ${ }^{4}$

The focus of health researchers should be on the individual and not on the disease. To start a clinical research, the researches need to formulate a question. The "questions" were divided into what, how, for what, what, 
why, where, and new attributes were also included such as who? for whom? how many? For the correct formulation of the question, which will generate the entire process of research development, the following was used: "When you do not know where you are going, any path leads you anywhere".

In addition, two premisses need to be on the researcher's mind: explain the formulation of a question and describe the title formulation. ${ }^{5}$ It is also suggested, based on the new focus of clinical research with humans, to predict the possibility of being a possible source (Randomized Clinical Trial) of protocols for systematic reviews (SR). The goal is to seek conclusions based on scientific evidence to guide researchers, professors, clinical professionals, and, most importantly, clients, in the case of Systematic Reviews that follow the Cochrane Collaboration model. ${ }^{6}$

In this sense, fundamental elements are configured for the planning of a relevant clinical study, in addition to defining the participants, the comparisons, the type of study, the potential benefits of the study for the research subjects and the types of clinical outcomes. . $^{6,7,89}$

The golden rule for comparing clinical treatments in humans is always emphasizing the search for clinically relevant outcomes (also the importance of focusing studies and results on the individual, on the client). However, it is not an easy task to plan, recruit, fund and develop controlled, randomized, crossover clinical studies - especially the desirable multi-center studies that can last for years of hard work. Therefore, it is increasingly sought to use surrogate clinical outcomes for the development of clinical studies, especially those in phase II, and eventually, after evaluation of the results obtained, it is logical and ethical to attempt a correlation with the primary outcomes by statistical methods, and with the use of validated endpoints in the literature.

Surrogate clinical outcomes have already been used in medicine, including many studies that validate surrogate clinical outcomes, thus increasing their value and acceptance., ${ }^{7,10,11}$

There remains a greater discussion on the use of secondary clinical outcomes, or surrogates, or substitutes, in clinical dental practice. Therefore, scientific research should seek even greater basing and validation of these outcomes so that they can be used, in particular in the development of techniques and drugs in Phase II studies, always obeying ethics and good research practices in humans. Thus, this paper aims to conceptualize clinical endpoints as well as critically assess when surrogate endpoints can and when they should not be used to the detriment of clinical studies aimed at primary or clinically relevant clinical outcomes.

\section{Methodology}

The articles were searched in databases such as PubMed, Cochrane Library, SciELO, EMBASE, from 1980 to 2019, using the keywords "surrogate endpoint", "surrogates outcomes", and "Randomized Clinical Trials". A critical and conceptual evaluation of the importance of the use of surrogate endpoints in dentistry, methods of validating the results, as well as the necessary care for their use, were analyzed, discussed and, consequently, some conclusions or indicatives of uses and limitations were proposed.

\section{Results}

\section{Stating the types of outcomes in clinical research}

The outcome of a clinical trial should necessarily meet three criteria: a) Be interpretable and measurable; b) Be sensitive to the purpose of the study and; c) Be clinically relevant, ${ }^{7,9}, 9$ In clinical research, the main goals are to address the relevant clinical outcomes, main or real, defined as follows: "A hard clinical outcome (primary) directly measures clinical key benefits for the participants, for example, survival or reduction of the effect of a disease".,11 Primary clinical result of a research is the "true impact of the disease on the patient's life". The patient feels about the outcome of the research, is morbidity (quality of life) or mortality (survival time). Primary outcomes are events, variables or experiences that are measured, because it is supposed to be influenced by the type of intervention under study. The expected outcome that was used by the investigator to calculate the 
sample size (number needed to treat - NNT) and/ or determine the effects of the interventions, was applied in phase III clinical studies. Finally, the findings, outcomes, endpoints, "results" observed at the end of the study. ${ }^{5,711,13,13}$

Randomized controlled trials (RCTs) are the most reliable way to obtain answers about the efficacy and safety of interventions in clinical practice. An ideal primary outcome should examine a clear and compelling benefit to the patient (subject, research subject). Final endpoints such as myocardial infarction, stroke, hospitalizations for heart failure (morbidity), and death (mortality) are clinically relevant and should be considered in clinical trials evaluating therapies with broad application potential as exemplified in Table 1.5,7,10,12,14

The conceptualization of a surrogate outcome may thus be defined: "A surrogate outcome is a laboratory measure or signal or physical symptom used as a substitute for a clinically relevant outcome, which measures directly how a participant feels, acts, or survives. It is what "the patient feels, reports or presents". Thus, it is desirable that the changes produced by a therapy in a surrogate outcome reflect the changes in a clinically meaningful outcome, there must be an association between the response by surrogate measures and the response by true clinical outcomes, only with the bias temporal 7,8,11,12,13,15,16,17.

In the absence of a table summarizing the specific clinical outcomes for dentistry, based on the literature searched in relevant clinical studies specific to each clinical situation and/or disease, we elaborated Table 2. Several types of outcomes can be evaluated in clinical studies with new or existing drugs, for example. We must consider that in addition to its advantages and disadvantages, there is a hierarchy in the levels of clinical evidence provided by outcomes: ${ }^{5,18-19,20,21}$

Level 1: A direct measure of clinical benefit (i.e., a major outcome);

Level 2: A validated substitute outcome;

Level 3: A substitute outcome not validated, but which is reasonably capable of indicating clinical benefit and;

Level 4: A correlation that is only measure of biological activity, unrelated to clinical benefit.

This finding is essentially important because, as a rule, the pharmaceutical industry usually tries (and succeeds) in convincing physicians on the use of substances of unproven clinical benefit, using only arguments of surrogate outcomes. This is very common with antihypertensive, diuretic, as was with thalidomide, with the first non-steroidal antiinflammatory specific COX-2 inhibitors, just to name a few examples. ${ }^{12,20,22,34,37,44}$

On the other hand, it is undeniable that clinical trials with hard endpoints require: a) High Costs; b) Long development time; c) Complex planning and organization; d) Large sample size; e) Long follow-up time $^{7}$ and we dare to add f). Ethical considerations (the subject of the research, the individual as the research center, increasing risks of unwanted side effects).

Table 1. Some samples of true endpoints and surrogate endpoints in medicine.

\begin{tabular}{lcc}
\hline Research condition & True endpoints & Surrogate endpoints \\
\hline Hypertension & Cardiovascular events & Arterial pressure \\
Cancer & Death & Tumor size \\
Diabetes & Cardiovascular events & Glycaemia \\
Alzheimer disease & Death/functional evaluation & Brain image exam \\
Vaccine & Disease protection & Serological response \\
Osteoporosis & Bone fracture & Bone density \\
Short & Final height of the individual & Height gain \\
Glaucoma & Loss of vision & Intraocular pressure \\
Atherosclerosis & Cerebral infarction & Arterial pressure
\end{tabular}

Modified from Coutinho, ${ }^{7}$ Piantadosi ${ }^{10}$ and Karberg and Speers. ${ }^{11}$ 
Table 2. Some samples of True Endpoints and Surrogate Endpoints in Clinical Dentistry.

\begin{tabular}{lcc}
\hline Research condition & True endpoints & Surrogate endpoints \\
\hline Dental caries & Pain & Thermal and/or electrical testing \\
Ceriodontal disease & Dental loss & Clinical attachment level \\
& Pain & Bleeding on probing \\
Pulpitis & Dental loss & Radiographic control \\
& Pain & Absence of clinical symptomatology \\
Anesthesia & Pressure & Clinical signs/symptoms \\
& Cure & Degree of satisfaction (questionnaires) \\
Herpes simplex T-1 & Absence of fear
\end{tabular}

Constructed based on the specific literature for each condition/disease researched in the main databases, and in the clinical practice of the authors of this work.

\section{Surrogate endpoints in dental clinical research}

As previously discussed, a surrogate outcome is information obtained in a complementary examination, which the patient does not feel. When doing a carotid Doppler, it can demonstrate atherosclerosis. If the patient is asymptomatic, this observed atherosclerosis is a substitute outcome of a future stroke that may occur, even if there is no guarantee. It is therefore a prediction. Similarly, there is no guarantee that a treatment that improves this atherosclerosis will reduce the likelihood of stroke. ${ }^{11,13,22}$

Surrogate clinical outcomes can occur in two ways, either they are predicted (planned) prior to the start of a study, or is information that comes up during the study (unplanned), and is used to evaluate "additional intervention effects". A surrogate outcome may involve the same event, variable or experience of the primary outcome, but measured over a different period. The surrogate outcome may also be related to different events, variables, or experiences. They are usually measures of the disease process and are considered "surrogate markers" or "intermediate measures" where results are usually laboratory measurements, clinical measurements (such as periodontal probing) or imaging studies (radiographs, models, CT scans) a clinical event of interest. $5,7,11,22,23$

\section{Discussion}

\section{Surrogate endpoints: when to use?}

Substitute outcomes are used because they can: i. Be measured earlier; ii. Generally are convenient or less invasive, and iii. Can accelerate the approval process of a new technique, a new drug, a new material (in our case, dental). Additional advantages are: their use may likely reduce the sample size of clinical trials, shorten its duration, and thus reduce its cost. Using surrogate endpoints also exposes fewer study participants to the risk of adverse reactions to the study product. There are a number of examples of surrogate endpoints in clinical trials, including dentistry. ${ }^{23,24,25,26}$ In the present study, the results of the present study are presented in Table 1 and Table 2.

As a rule, when a clinically relevant outcome is used, it is adopted in the final confirmatory clinical study, with a larger " $\mathrm{n}$ " sample of patients (phase III) of a new health therapy, while a surrogate outcome is mostly often used in initial exploratory studies (phases I and II) of the study product or therapy. 
The regulatory authorities (ANVISA-Brazil, CDCUSA and WHO) may require the use of a primary clinical outcome, rather than a surrogate clinical outcome, as the most important health indicator in clinical studies for a specific disease. Such events are rare because many individuals need to participate in confirmatory studies. However, in the initial exploratory phase of a new therapy, it is common to use a surrogate outcome, since it reduces the sample size, the duration of the study and, considering the source of research funding, substantially lower costs.7

For example, in studies of cardiovascular problems, blood pressure and cholesterol levels are commonly used as surrogate measures, whereas true clinical outcomes are myocardial infarction and death. ${ }^{8}$

In the case of dentistry, for the study of a new type of intervention to treat periodontal diseases, where the clinically relevant outcome would be dental loss, one can use the following surrogate endpoints: a) clinical attachment level; b) Bleeding on probing and c) probing depth. In order to evaluate a new dental implant design, or even a new implant and abutment material, the clinically relevant outcome would be failure (absence of osseointegration) and implant loss, whereas the following surrogate outcomes can be evaluated initially, with lower costs and follow-up time: a) survival rate of implants; b) clinical and radiographic controls and c) periimplant bone loss. ${ }^{13,23,27,28}$

In addition, in interdisciplinary and even transdisciplinary work, the use of surrogate outcomes has gained important ground. The association between gingivitis and periodontitis as chronic oral diseases and brain diseases affecting small blood vessels (stroke, dementia, Alzheimer's disease) was studied by Aarabi et al. ${ }^{29}$ With the use of surrogate endpoints (magnetic resonance imaging, evidence of cerebrovascular ischemia, acute ischemia and stroke), researchers have found evidence of worsening of this pathogenesis when periodontal diseases are associated. They suggest, however, large cohort studies to be able to further substantiate this correlation.

In a systematic review supplemented with meta-analysis, there were studied in the elderly (>65 years): a) prevalence of endodontically treated teeth; b) prevalence of periapical lesions in non- endodontically treated teeth; c) prevalence of radiolucency in endodontically treated teeth using periapical radiographs (periapical radiolucency as surrogate). This type of outcome made RS possible with MA because it was a prevalence and non-incidence study, which in this case would be a major outcome. ${ }^{30}$

Measurements of breath expelled by volunteers have been studied as possible surrogates for examination of blood samples from individuals with systemic diseases such as diabetes mellitus. The ketone odor characteristic of the air expelled by people with diabetes (beta-hydroxybutyrate / acetoacetate and acetone) produced by the body to supply the lack of energy sources in cases of glucose shortage was measured. Thus, it is possible to avoid the use of traumatic methods such as needles and blood collection devices for glycemic dosages. ${ }^{24,25,31}$

In particular, periodontal literature has been discussing the use of secondary endpoints (measurement of clinical attachment loss or clinical probing depth) as substitutes or surrogates for tooth loss due to periodontal disease. However, substitute outcomes are not always found for other areas of dentistry. Thus, researchers developed a new tool, called "clinical success parameters" (CSP), based on three clinical performance criteria: a) biological parameters; b) technical parameters; and c) quality of life parameters, to be used in pre, trans and posttreatment, with fulcrum in calculations of changes in follow-up and the use of weight of the findings, with a focus on the professional, patients, technical and biological aspects. ${ }^{13}$

\section{Surrogate endpoints: when I should not use them?}

Because surrogate or intermediate outcomes are laboratory, imaging, clinical or physiological (functional) parameters, they generally do not have statistical power to evaluate clinically relevant outcomes. Thus, in the antihypertensive treatment, reduction of the P.A. is a substitute outcome of the reduction of stroke (clinical outcome). Gain in the clinical attachment level is a surrogate outcome, while dental loss is a major clinical outcome.,13

Even if there is a solid pathophysiological basis, or by confirmations of images ( $x$-rays, scans, study 
models), they should not serve as the basis for decision making in clinical practice. There are a number of examples in medicine and dentistry that support this assertion and should alert us to be careful in making therapeutic decisions based on studies using surrogate endpoints. $13,17,32,33,34,35$

Thus, Lee ${ }^{28}$ proposed the need to evaluate and validate surrogate endpoints for peri-implantitis clinical studies (already questioned), since most of the true outcomes (i.e., implant failure) preferred in these studies hampers the establishment of therapies for the treatment of peri-implantitis, because implant failure data are presented only as a consequence of therapy and not as an objective of an investigation. Thus, the author reports that surrogate outcomes such as: a) average probing depth of the peri-implant pocket; b) mean level of clinical attachment on the implant and; c) average bleeding on probing is still little mentioned in the literature, which does not yet provide evidence-based robustness that should be confirmed by long-term studies. The need to validate surrogate endpoints as an alternative to clinically relevant outcomes has also been advocated and encouraged by Buyse et al., ${ }^{18}$ through meta-analyzes of randomized clinical trials.

A literature review of four types of outcomes was carried out in randomized clinical trials on the treatment of periodontitis: a) typical number of outcomes per randomized clinical trials (RCT); b) the proportion of RCTs using the same outcome; c) the proportion of RCTs using true outcomes and; d) if the choice of treatment influenced the choice of outcome. The authors concluded that the use of multiple surrogate outcomes led to false-positive and false-negative conclusions, and that the selection and validation of outcomes in RCTs may be an important element in the resolution of controversies over periodontal treatments. ${ }^{23,27}$

Surrogate outcomes that allow predicting the effect of clinically relevant treatment on the outcome of interest are important when it is difficult or expensive to measure the primary outcome. However, there are situations in which the effect of the treatment on the surrogate outcome is positive, the surrogate and the result are correlated in a very positive way, but the treatment effect on the primary outcome is negative.
This is the phenomenon called the "substitution effect paradox" ${ }^{36}$

Infective endocarditis (IE/EBSA) still raises many controversies regarding the use or not of antibiotic prophylaxis, both from the point of view of dentists and cardiologists. A systematic review with very careful meta-analysis was developed to try to remedy the dilemma, since guidelines for cardiologists and dentists advised restricting antibiotic prophylaxis to high-risk groups (in Europe and the USA) or were against their use (in the United Kingdom-UK). The authors concluded, however, that the current evidence base for antibiotic prophylaxis use is limited, heterogeneous, and the methodological quality of many studies is poor, and note that post-procedure bacteremia is not a good substitute endpoint for IE/EBSA. ${ }^{33}$

The search for effective cancer treatment should allow patients to have a better or longer life and ideally both as clinical trial outcomes. Substitute outcomes such as time to progression or treatment of the disease may be employed to identify preliminary benefits. Nevertheless, their true validity as surrogate outcomes is questionable because many agents are involved, such as regulatory agencies, clinical professionals, the drug industry and, of course, and most importantly, patients and their families. Thus, it is suggested that clinical studies and systematic reviews should incorporate the parties involved to assess what the future holds for the outcomes and the type of clinical trial. ${ }^{21}$

In practical terms, the approval of new products under study with reference to the effects on a substitute outcome involves an extrapolation of the experience with commercial products an experimental product not yet tested. There have been several cases where treatments with a highly positive effect on a proposed substitute have turned out to be detrimental in true clinical outcomes. On the other hand, there are cases of treatments that provide clinical benefits, but with no measurable impact on the proposed substitutes. $7,8,11,37$

The use of surrogate endings allows the work to have a shorter follow-up, smaller sample size and cost reduction. It is ideal for phase I and phase II RCTs when it comes to the safety of an intervention, 
but it is inadequate when it comes to the conduct of action that will influence the lives of many phase III patients. This is because physiological variables do not guarantee the achievement of hard outcomes. ${ }^{38}$

Observing a set of volatile markers through expired air measurements (true halitosis), may allow the recognition and diagnosis of complex diseases, such as lung or breast cancers, and have been considered for studies as surrogate endpoints. However, due to technical problems of sampling and analysis, in addition to the lack of standardization, there are enormous variations between the results of the different studies already carried out, which is why the analysis of breath expelled has not yet been introduced in clinical practice, as routine of substitute outcome. ${ }^{24}$

Grimes and Schulz ${ }^{22}$ have evaluated some dangerous discrepancies between surrogate endpoints and clinically relevant outcomes in clinical studies, where consecrated drugs devoted to osteoporosis, cardiac arrhythmia and cardiovascular disease treatments in postmenopausal women, what actually occurred in the patients involved in the studies was an increase in the prevalence of bone fractures, increased mortality and even sudden death. The authors cited even that the maximum clinical: "A difference to be a difference must make a difference" also applies in clinical research.

Until the 1980s, experiences with a drug practically ran out even before it was released. However, after tragedies such as the one with thalidomide, health authorities began to require manufacturers to be more rigorous in controlling the safety of their medicines even after their commercialization. ${ }^{39}$

Unfortunately, in order to guarantee a few more years of exclusivity over the patent for lucrative drugs (Vioxx case), laboratories have begun to seek new indications for them. The case of Rofecoxib (Vioxx) was emblematic. With the discovery (surrogate or secondary outcome), that in addition to its proven efficacy in the treatment of arthritis, the drug also reduced intestinal polyps, and was also prescribed for the relief of various types of pain, such as menstrual cramps, toothache, myalgia, and migraine, this advanced its launch in the market. However, the drug was withdrawn from the market by the industry itself responsible for its production, since it is believed that between 88,000 and 140,000 cases of severe heart disease have resulted from long-term use of the drug. ${ }^{40,41}$

This true tragedy occurred mainly due to the delay in the discovery of the adverse effect and its relation with the drug, due to the inexistence of randomized, controlled, large, long-term and multicentric studies that aimed to evaluate the cardiovascular effects of specific inhibitors of COX-2. ${ }^{41,42,43,44}$

For generations, dental students were instructed to train clinical skills using their classmates as surrogate patients. These skills include verbal and communication skills (obtaining medical and social histories - anamnesis), as well as practices such as administration of local anesthetic by injection. Largely, such practices within dental education were accepted through "conventions." The question is whether the use of students in dental education as surrogate patients can create issues related to free and informed consent, coercion and confidentiality, and whether this type of behavior is professional. ${ }^{26,45}$

After extensive review of the current literature and discussion of research methodologies in clinical trials in dentistry, and in the absence of specific guidelines for the correct and rational use of surrogate outcomes in clinical practice, a flowchart is suggested to guide good practices in clinical research in dentistry. The goal is taking the patient as the center of research, considering health holistically, in the inseparability of physical, mental, social and spiritual well-being (Figure).

\section{Conclusions}

Based on the current literature stipulated for this research, we can conclude favorably to the use of the secondary endings in the following situations:

a. Substitute outcomes may play an important role in the initial drug development process due to shorter follow-up time and sample size, as well as the simpler methodology to demonstrate the effect of the intervention on important patient outcomes;

b. Evidence on the effects of an intervention based on surrogate outcomes usually precedes, with 


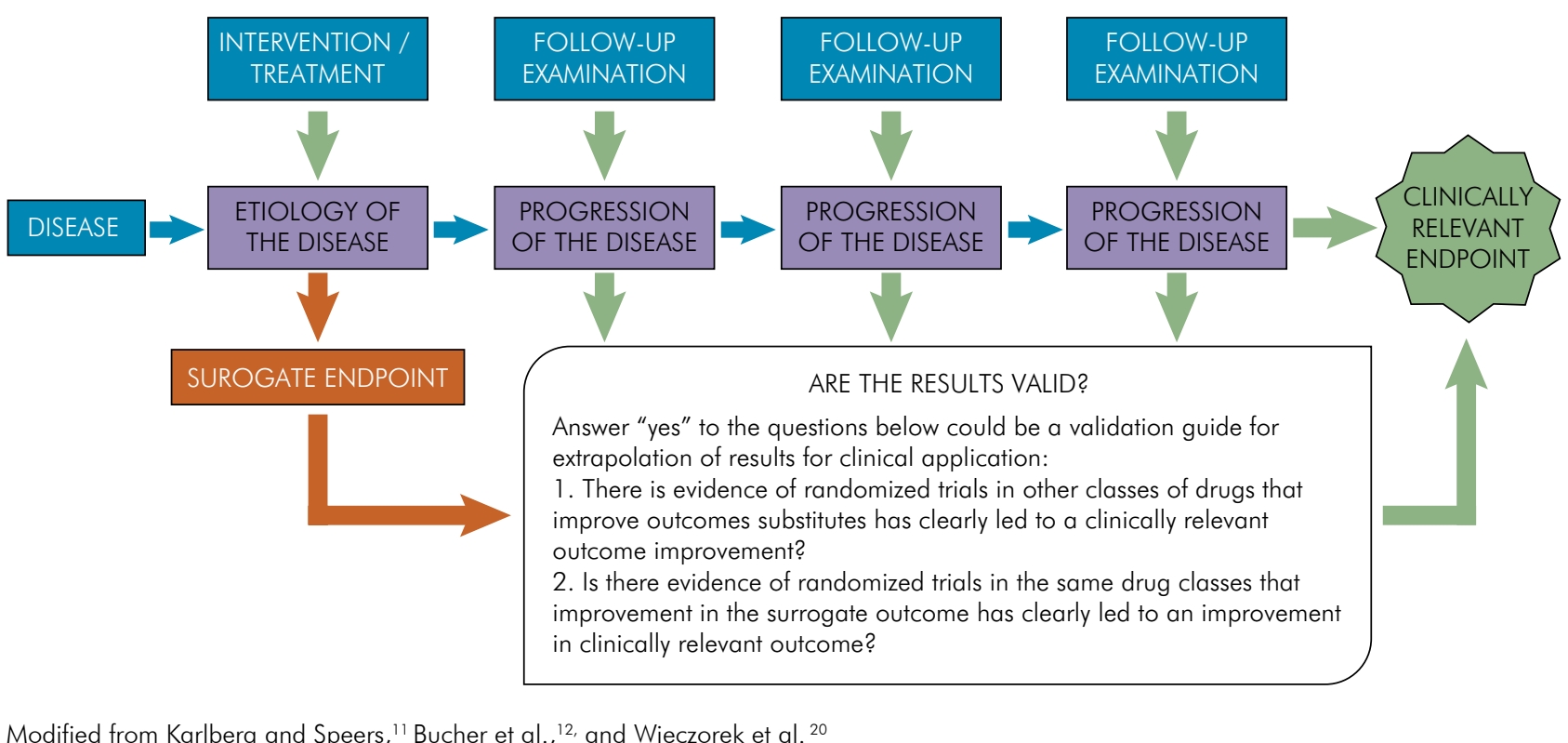

Figure. Proposal of a flow chart of actions for validation of surrogate endpoints for application in clinical dentistry

less risk of side effects and lower cost, clinically relevant outcomes for the patient;

c. The proven association between a surrogate outcome and a (relevant) major clinical outcome for the patient is essential to draw appropriate therapeutic conclusions, provided there is validation of scientific methods and surrogate outcomes.

We can also conclude by suggesting very careful about the use of secondary outcomes due to the following facts:

a. Thousands of lives may be lost unnecessarily because of reliance on non-RTC-validated surrogate markers for clinically relevant outcomes;

b. The use of surrogate outcomes, even if validated, may waste resources, provide ambiguous evidence and may not be extrapolated to other populations (absence of multicenter). They can also generate bias due to the individual interpretation of each researcher;

c. The use of surrogates that arise during the study (those unplanned) should be weighed and done with great caution; it is not prudent to anticipate the release of a new drug without the necessary time tracking.

\section{Acknowledgments}

The authors would like to thank the Pro-Rector of Research of University of Sao Paulo, and to the Research Committees of the Faculty of Dentistry of the University of São Paulo (CPq-FO), Bauru School of Dentistry (CPq-FOB) and School of Dentistry of Ribeirao Preto (CPq-FORP) and the Organizing Committee for participating in the "Grandes Desafios em Pesquisa Clinica na Odontologia" event.

\section{References}

1. World Health Organization. Preamble to the Constitution of the World Health Organization as adopted by the International Health Conference, New York, 19-22 June, 1946; signed on 22 July 1946 by the representatives of 61 States (Official Records of the World Health Organization, no. 2, p. 100) and entered into force on 7 April 1948 [cited 2019 May 22]. Available from: http://www.who.int/ governance/eb/who_constitution_en.pdf

2. Vader JP. Spiritual health: the next frontier. Eur J Public Health. 2006 Oct;16(5):457. https://doi.org/10.1093/eurpub/ckl234 
3. Nagase, M. Does a multi-dimensional concept of health include spirituality? Analysis of Japan Health Science Council's Discussionson WHO's 'Definition of Health' (1998). Int J Appl Sociol. 2012;2. https://doi.org/10.5923/i.ijas.20120206.03

4. Reis VM, Videira AA. [John Ziman and post-academic science: consensibility, consensus, and reliability]. Sci Stud. 2013;11(3):583-611. Portuguese. https://doi.org/10.1590/S1678-31662013000300007

5. Ferreira JC, Patino CM. Types of outcomes in clinical research. J Bras Pneumol. 2017 jan/fev;43(1):5. https://doi.org/10.1590/s1806-37562017000000021

6. Smith R, Chalmers I. Britain's gift: a "Medline" of synthesised evidence. BMJ. 2001 Dec;323(7327):1437-8. https://doi.org/10.1136/bmi.323.7327.1437

7. Coutinho MSSA. [Surrogate and relevant clinical endpoints. What are they and how to interpret them?] Rev Bras Hipertens. 2002 Jan/Mar; (1):24-8. Portuguese.

8. Coutinho ES, Cunha GM. [Basic concepts in epidemiology and statistics for reading controlled clinical trials]. Br J Psychiatry. 2005 Jun;27(2):146-51. Portuguese. https://doi.org/10.1590/S1516-44462005000200015

9. Kane R. Understanding health care outcomes research. Burlington: Jones \& Bartlett Learning; 2006.

10. Piantadosi S. Clinical trials: a methodologic perspective. Hoboken: John Wiley \& Sons; 2017.

11. Karlberg JP, Speers MA, editors. Revisão de estudos clínicos: um guia para o comitê de ética. Hong Kong: Karlberg, Johan Petter Einar, 2014.

12. Bucher HC, Guyatt GH, Cook DJ, Holbrook A, McAlister FA. Users' guides to the medical literature: XIX. Applying clinical trial results. A. How to use an article measuring the effect of an intervention on surrogate end points. JAMA. 1999 Aug;282(8):771-8. https://doi.org/10.1001/jama.282.8.771

13. Just B, Quass S, Rudolph H, Waler MH, Foest EM, Luthardt RG. A novel surrogate parameter measuring the outcome in dental clinical trials. Res Ver J Dent Sci. 2017 Feb;5(1):70-5.

14. Piantadosi S. Clinical trials: a methodologic perspective. Hoboken: Wiley; 2013.

15. Ellenberg S, Hamilton JM. Surrogate endpoints in clinical trials: cancer. Stat Med. 1989 Apr;8(4):405-13. https://doi.org/10.1002/sim.4780080404

16. Prentice RL. Surrogate endpoints in clinical trials: definition and operational criteria. Stat Med. 1989 Apr;8(4):431-40. https://doi.org/10.1002/sim.4780080407

17. Fleming TR, DeMets DL. Surrogate end points in clinical trials: are we being misled? Ann Intern Med. 1996 Oct;125(7):605-13. https://doi.org/10.7326/0003-4819-125-7-199610010-00011.

18. Buyse M, Molenberghs $G$, Burzykowski T, Renard D, Geys $H$. The validation of surrogate endpoints in meta-analyses of randomized experiments. Biostatistics. 2000 Mar;1(1):49-67. https://doi.org/10.1093/biostatistics/1.1.49

19. U.S. Department of Health and Human Services. Food and Drug Administration. Clinical Trial endpoints for the approval of cancer drugs and biologics: guidance for industry. Silver Springer: Food and Drug Administration; 2018 [cited 2019 May 22]. Available from: http://www.fda.gov/downloads/Drugs/Guidances/ucm071590.pdf

20. Wieczorek A, Rys P, Skrzekowska-Baran I, Malecki M. The role of surrogate endpoints in the evaluation of efficacy and safety of therapeutic interventions in diabetes mellitus. Rev Diabet Stud. 2008;5(3):128-35. https://doi.org/10.1900/RDS.2008.5.128

21. Wilson MK, Karakasis K, Oza AM. Outcomes and endpoints in trials of cancer treatment: the past, present, and future. Lancet Oncol. 2015 Jan;16(1):e32-42. https://doi.org/10.1016/S1470-2045(14)70375-4

22. Grimes DA, Schulz KF. Surrogate end points in clinical research: hazardous to your health. Obstet Gynecol. 2005 May;105(5 Pt 1):1114-8. https://doi.org/10.1097/01.AOG.0000157445.67309.19

23. Hujoel PP, DeRoven TA. A survey of endpoint characteristics in periodontal clinical trials published 1988-1992, and implications for future studies. J Clin Periodontol. 1995 May;22(5):397-407. https://doi.org/10.1111/i.1600-051X.1995.tb00167.x

24. Miekisch W, Schubert JK, Noeldge-Schomburg GF. Diagnostic potential of breath analysis_focus on volatile organic compounds. Clin Chim Acta. 2004 Sep;347(1-2):25-39. https://doi.org/10.1016/i.cccn.2004.04.023

25. O'Hara ME, Clutton-Brock TH, Green S, Mayhew CA. Endogenous volatile organic compounds in breath and blood of healthy volunteers: examining breath analysis as a surrogate for blood measurements. J Breath Res. 2009 Jun;3(2):027005. https://doi.org/10.1088/1752-7155/3/2/027005

26. Rosenberg M, Orr DL 2nd, Starley ED, Jensen DR. Student-to-student local anesthesia injections in dental education: moral, ethical, and legal issues. J Dent Educ. 2009 Jan;73(1):127-32.

27. Faggion CM Jr, Listl S, Tu YK. Assessment of endpoints in studies on peri-implantitis treatment-a systematic review. J Dent. 2010 Jun;38(6):443-50. https://doi.org/10.1016/i.jdent.2010.03.003

28. Lee DW. Validated surrogate endpoints needed for peri-implantitis. Evid Based Dent. 2011;12(1):7. https://doi.org/10.1038/si.ebd.6400770

29. Aarabi G, Thomalla G, Heydecke G, Seedorf U. Chronic oral infection: an emerging risk factor of cerebral small vessel disease. Oral Dis. 2019 Apr;25(3):710-9. https://doi.org/10.1111/odi.12912 
Surrogate endpoints: when to use and when not to use? A critical appraisal of current evidences

30. Hamedy R, Shakiba B, Pak JG, Barbizam JV, Ogawa RS, White SN. Prevalence of root canal treatment and periapical radiolucency in elders: a systematic review. Gerodontology. 2016 Mar;33(1):116-27. https://doi.org/10.1111/ger.12137

31. Gokdogan O, Catli T, Ileri F. Halitosis in otorhinolaryngology practice. Iran J Otorhinolaryngol. 2015 Mar;27(79):145-53.

32. ALLHAT Collaborative Research Group. Major cardiovascular events in hypertensive patients randomized to doxazosin vs chlorthalidone: the antihypertensive and lipid-lowering treatment to prevent heart attack trial (ALLHAT). JAMA. 2000 Apr;283(15):1967-75. https://doi.org/10.1001/jama.283.15.1967

33. Cahill TJ, Harrison JL, Jewell P, Onakpoya I, Chambers JB, Dayer M, et al. Antibiotic prophylaxis for infective endocarditis: a systematic review and meta-analysis. Heart. 2017 Jun;103(12):937-44. https://doi.org/10.1136/heartinl-2015-309102

34. Cardiac Arrhythmia Suppression Trial (CAST) Investigators. Preliminary report: effect of encainide and flecainide on mortality in a randomized trial of arrhythmia suppression after myocardial infarction. N Engl J Med. 1989 Aug;321(6):406-12. https://doi.org/10.1056/NEJM198908103210629

35. Baker DW. Reading between the lines: deciphering the connections between literacy and health. J Gen Intern Med. 1999 May;14(5):315-7. https://doi.org/10.1046/j.1525-1497.1999.00342.x

36. Vanderweele TJ. Surrogate measures and consistent surrogates. Biometrics. 2013 Sep;69(3):561-9. https://doi.org/10.1111/biom.12071

37. Holloway RG, Dick AW. Clinical trial end points: on the road to nowhere? Neurology. 2002 Mar;58(5):679-86. https://doi.org/10.1212/WNL.58.5.679

38. Korman M, Weiss PL. Living labs: overview of ecological approaches for health promotion and rehabilitation. Disabil Rehabil. 2015 July;38(7):1-7. https://doi.org/10.3109/09638288.2015.1059494

39. Dally A. Thalidomide: was the tragedy preventable? Lancet. 1998 Apr;351(9110):1197-9. https://doi.org/10.1016/S0140-6736(97)09038-7

40. Fitzgerald GA. Coxibs and cardiovascular disease. N Engl J Med. 2004 Oct;351(17):1709-11. https://doi.org/10.1056/NEJMp048288

41. Araujo LF, Soeiro AM, Fernandes JL, Serrano Júnior CV. Cardiovascular events: a class effect by COX-2 Inhibitors. Arq Bras Cardiol. 2005 Sep;85(3):222-9. https://doi.org/10.1590/S0066-782X2005001600016

42. Bombardier, C. et al. Comparison of upper gastrointestinal toxicity of rofecoxib and naproxen in patients with rheumatoid arthritis. VIGOR Study Group. The New England journal of medicine 343, 1520-1528, 1522 p following 1528, (2000). https://doi.org/10.1056/NEJM200011233432103

43. Topol EJ. Failing the public health—rofecoxib, Merck, and the FDA. N Engl J Med. 2004 Oct;351(17):1707-9. https://doi.org/10.1056/NEJMp048286

44. Psaty BM, Furberg CD. COX-2 inhibitors-lessons in drug safety. N Engl J Med. 2005 Mar;352(11):1133-5. https://doi.org/10.1056/NEJMe058042

45. Holden A. The use of students as surrogates: the ethical, legal and professional issues of student-student practical learning in dental education. J Law Med. 2018 Feb;25(2):380-7. 